\title{
Mass formulas and thermodynamic treatment in the mass-density-dependent model of strange quark matter
}

\author{
G. X. Peng, ${ }^{1,2,4}$ H. C. Chiang, ${ }^{2}$ J. J. Yang, ${ }^{3}$ L. Li, ${ }^{4}$ and B. Liu ${ }^{2}$ \\ ${ }^{1}$ China Center of Advanced Science and Technology (World Laboratory), Beijing 100080, China \\ ${ }^{2}$ Institute of High Energy Physics, Academia Sinica, Beijing 100039, China \\ ${ }^{3}$ Department of Physics, Nanjing Normal University, Nanjing 210024, China \\ ${ }^{4}$ Department of Physics, Nankai University, Tianjin 300071, China
}

\begin{abstract}
The previous treatments for strange quark matter in the quark mass-density-dependent model have unreasonable vacuum limits. We provide a method to obtain the quark mass parametrizations and give a self-consistent thermodynamic treatment which includes the MIT bag model as an extreme. In this treatment, strange quark matter in bulk still has the possibility of absolute stability. However, the lower density behavior of the sound velocity is opposite to previous findings.
\end{abstract}

PACS numbers: 24.85.+p, 12.38.Mh, 12.39.Ba, 25.75.-q

\section{INTRODUCTION}

Since Witten's conjecture that quark matter with strangeness per baryon of order unity might be bound [1], an extensive body of literature has investigated the stability and/or probabilities of strange quark matter (SQM) 22]. Because the application of perturbative quantum chromodynamics (QCD) to strong-coupling domain is unbelievable while the lattice approach is presently limited to the case of zero chemical potential, we have to resort to phenomenological models. One of the most famous models is the MIT bag model with which Farhi and Jaffe find that SQM is absolutely stable around the normal nuclear density for a wide range of parameters [3]. Further investigations have also been carried out by

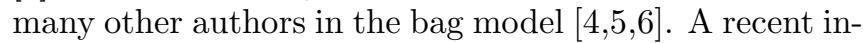
vestigation indicates a link of SQM to the study of quark condensates [7] while a more recent work has carefully studied the relation between the charge and critical density of SQM [8].

Chakrabarty et al. [9, 10] have discussed the limitation of the conventional MIT bag model which assumes that the quarks are asymptotically free within the bag. In order to incorporate the strong interaction between quarks, one way is to fall back on the perturbation theory, which is questionable in the strong-coupling domain. An alternative way is to make the quark masses densitydependent. In this nonperturbative treatment, the strong interaction between quarks is mimicked by the proper variation of quark masses with density. There are two questions of crucial importance to this model. One is how to parametrize quark masses, the other concerns thermodynamic treatment. However, the two aspects are not self-consistent in literature presently.

Here are the popularly used parametrizations for quark masses $m_{q}(q=u, d, s$,$) :$

$$
\begin{aligned}
m_{u, d} & =\frac{B}{3 n_{b}}, \\
m_{s} & =m_{s 0}+\frac{B}{3 n_{b}},
\end{aligned}
$$

where $m_{s 0}$ is the $s$ quark current mass, $n_{b}$ is the baryon number density, $B$ is the famous MIT bag constant. Equation (11) was first used to study light quark matter [11], and later extended to Eq. (2) to investigate strange quark matter $09.10,12$.

As for the thermodynamic treatment, there exist two controversial ones in literature up to now. One expresses the total pressure of SQM as 9, 10

$$
P_{1}=-\Omega,
$$

where $\Omega$ is the ordinary thermodynamic potential density of SQM [see Eq. (40)]. The other adopts the following expression [12]:

$$
P_{2}=-\Omega+n_{b} \frac{\partial \Omega}{\partial n_{b}} .
$$

The extra term in Eq. (位) is said to arise from the baryon density dependence of quark masses. This difference leads to significantly different results. Therefore, it is meaningful to take a check of the two thermodynamic treatments.

As is well known, the QCD vacuum is not necessarily empty. To obtain the vacuum properties, let us take the limit $n_{b} \rightarrow 0$ for the two treatments. It is easy to obtain, at zero temperature, the limits

$$
\begin{aligned}
& \lim _{n_{b} \rightarrow 0} P_{1}=0, \\
& \lim _{n_{b} \rightarrow 0} E_{1}=B,
\end{aligned}
$$

for the first treatment, and the limits

$$
\begin{aligned}
& \lim _{n_{b} \rightarrow 0} P_{2}=-B, \\
& \lim _{n_{b} \rightarrow 0} E_{2}=2 B,
\end{aligned}
$$

for the second treatment. Here $E_{1}$ and $E_{2}$ are the corresponding energy densities.

According to the fundamental idea of MIT bag model, QCD vacuum has a constant energy density $B$, the famous bag constant. The mass parametrization Eq. (1) 
is just obtained from this requirement $\left(\lim _{n_{b} \rightarrow 0} E_{1} \rightarrow\right.$ $3 m_{q} n_{b}$ for flavor-symmetric case) 11]. The constant vacuum energy comes from the fact that QCD vacuum must have a pressure to maintain pressure balance at the bag boundary. Obviously, the first treatment can give the correct vacuum energy and a wrong QCD vacuum pressure. On the contrary, the second treatment leads to the correct $\mathrm{QCD}$ vacuum pressure but a wrong vacuum energy. In fact, this is just caused by the ignorance of the QCD vacuum energy which guarantees the pressure balance at the bag boundary.

It should be pointed out that in getting the unreasonable limits (5)-(8), we have used the quark mass formulas (11) and (2). Because these formulas are pure parametrizations without any real support from underlying theories, one may ask if the contradictions can be solved by choosing other parametrizations? According to our present investigation, one should modify the quark mass formulas and thermodynamic treatment simultaneously.

It is the aim of this paper to give a self-consistent treatment which includes the conventional MIT bag model as an extreme. In our new treatment, strange quark matter in bulk still has the possibility of absolute stability. However, the lower density behavior of the sound velocity in SQM is opposite to previous findings.

In the following section, we first derive the new quark mass formulas and describe our thermodynamic treatment, and then in Sec. III, we present our results in studying SQM with this model. Section [V] is a short summary.

\section{FRAMEWORK}

Let us schematically write the QCD Hamiltonian density as

$$
H_{\mathrm{QCD}}=H_{k}+\sum_{q} m_{q 0} \bar{q} q+H_{\mathrm{I}},
$$

where $H_{k}$ is the kinetic term, $m_{q 0}$ is the quark current mass, and $H_{\mathrm{I}}$ is the interaction part. The summation goes over all flavors considered.

The basic idea of the quark mass-density-dependent model of strange quark matter is that the system energy can be expressed as the same form with a proper noninteracting system. The strong interaction between quarks is included within the appropriate variation of quark masses with density. In order not to confuse with other mass concepts, we refer such a density-dependent mass to an equivalent mass in this paper. Therefore, if we use the equivalent mass $m_{q}$, the system Hamiltonian density should be replaced by an Hamiltonian density of the form

$$
H_{\mathrm{eqv}}=H_{k}+\sum_{q} m_{q} \bar{q} q,
$$

where $m_{q}$ is the equivalent mass to be determined. Obviously, we must require that the two Hamiltonian densities $H_{\text {eqv }}$ and $H_{\mathrm{QCD}}$ have the same eigenenergy for any eigenstate $|\Psi\rangle$, i.e.,

$$
\left\langle\Psi\left|H_{\text {eqv }}\right| \Psi\right\rangle=\left\langle\Psi\left|H_{\mathrm{QCD}}\right| \Psi\right\rangle .
$$

Applying this equality respectively to the state $\left|n_{b}\right\rangle$ with baryon number density $n_{b}$ and the vacuum state $|0\rangle$, and then taking the difference, one has

$$
\left\langle n_{b}\left|H_{\text {eqv }}\right| n_{b}\right\rangle-\left\langle 0\left|H_{\text {eqv }}\right| 0\right\rangle=\left\langle n_{b}\left|H_{\mathrm{QCD}}\right| n_{b}\right\rangle-\left\langle 0\left|H_{\mathrm{QCD}}\right| 0\right\rangle .
$$

The simplest and most symmetric solution for the equivalent mass from this equation is

$$
\begin{aligned}
m_{q} & =m_{q 0}+\frac{\left\langle H_{\mathrm{I}}\right\rangle_{n_{b}}-\left\langle H_{\mathrm{I}}\right\rangle_{0}}{\sum_{q}\left[\langle\bar{q} q\rangle_{n_{b}}-\langle\bar{q} q\rangle_{0}\right]} \\
& \equiv m_{q 0}+m_{\mathrm{I}}
\end{aligned}
$$

where we have used the symbol definitions: $\left\langle H_{\mathrm{I}}\right\rangle_{n_{b}} \equiv$ $\left\langle n_{b}\left|H_{\mathrm{I}}\right| n_{b}\right\rangle,\left\langle H_{\mathrm{I}}\right\rangle_{0} \equiv\left\langle 0\left|H_{\mathrm{I}}\right| 0\right\rangle$, and $\langle\bar{q} q\rangle_{n_{b}} \equiv\left\langle n_{b}|\bar{q} q| n_{b}\right\rangle$, $\langle\bar{q} q\rangle_{0} \equiv\langle 0|\bar{q} q| 0\rangle$.

Therefore, if quarks are decoupled, they should take the equivalent mass of the form (13) to keep the system energy unchanged. From Eq. (13) we see that the equivalent mass $m_{q}$ includes two parts: one is the original mass or current mass $m_{q 0}$, the other is the interacting part $m_{\mathrm{I}}$. Because $m_{\mathrm{I}}$ equals to the ratio of the total interacting part of the energy density and the total relative quark condensate, it is flavor-independent and densitydependent. Because of the quark confinement and the asymptotic freedom, i.e.,

$$
\begin{aligned}
& \lim _{n_{b} \rightarrow 0} m_{\mathrm{I}}=\infty, \\
& \lim _{n_{b} \rightarrow \infty} m_{\mathrm{I}}=0,
\end{aligned}
$$

the reasonable form might be

$$
m_{\mathrm{I}}=\frac{D}{n_{b}^{z}}
$$

Accordingly, we have

$$
m_{q}=m_{q 0}+\frac{D}{n_{b}^{z}},
$$

where $D$ is a free parameter to be determined by stability arguments. Obviously, $z>0$ for confined particles and $z<0$ for nonconfined particles. In Eqs. (11) and (2), $z=1$. However, just as mentioned in the Introduction section, Eqs. (11) and (2) is closely linked to the first thermodynamic treatment, and thus unsuitable for our case. We now discuss the determination of $m_{\mathrm{I}}$ which is consistent with our thermodynamic treatment. 
Firstly, we express the interacting part of the energy density $\left\langle H_{\mathrm{I}}\right\rangle \equiv\left\langle H_{\mathrm{I}}\right\rangle_{n_{b}}-\left\langle H_{\mathrm{I}}\right\rangle_{0}$ [the numerator in Eq. [13] ] as

$$
\begin{aligned}
\left\langle H_{\mathrm{I}}\right\rangle & =\frac{1}{2 V} \iint_{V} v(r)\left(3 n_{b} d \overrightarrow{r_{1}}\right)\left(3 n_{b} d \overrightarrow{r_{2}}\right) \\
& =18 \pi n_{b}^{2} \int_{0}^{R} v(r) r^{2} d r,
\end{aligned}
$$

where $r=\left|\overrightarrow{r_{1}}-\overrightarrow{r_{2}}\right|, v(r)$ is the quark-quark interaction, $R$ is the SQM radius, $V=4 / 3 \pi R^{3}$ is the volume. The extra factor $1 / 2$ is responsible for double counting.

Because of the following obvious equality:

$$
\lim _{n_{b} \rightarrow 0} \frac{\langle\bar{q} q\rangle_{n_{b}}}{\langle\bar{q} q\rangle_{0}}=1,
$$

the Taylor series of the relative condensate at zero density has the following general form:

$$
\frac{\langle\bar{q} q\rangle_{n_{b}}}{\langle\bar{q} q\rangle_{0}}=1-\frac{n_{b}}{\rho_{q}^{\prime}}+\text { higher orders in } n_{b}+\cdots .
$$

If taking it only to first order approximation, we have

$$
\sum_{q}\left[\langle\bar{q} q\rangle_{n_{b}}-\langle\bar{q} q\rangle_{0}\right]=\sum_{q}\left[-\langle\bar{q} q\rangle_{0} / \rho_{q}^{\prime}\right] n_{b} \equiv A n_{b} .
$$

Taking the ratio of Eqs. 201 and (23), we get

$$
m_{\mathrm{I}}=\frac{18 \pi}{A} n_{b} \int_{0}^{R} v(r) r^{2} d r .
$$

According to the lattice calculation 13 and string model investigation [14], the quark-quark interaction is proportional to the distance, i.e., $v(r)=\alpha r$. We thus have

$$
m_{\mathrm{I}}=\frac{18 \pi \alpha}{A} n_{b} \frac{R^{4}}{4} \propto \frac{1}{n_{b}^{1 / 3}} .
$$

Therefore, we should take in Eq. (18) $z=1 / 3$, i.e.,

$$
m_{q}=m_{q 0}+\frac{D}{n_{b}^{1 / 3}},
$$

where $D$ is a parameter to be determined by stability arguments.

Because the Hamiltonian density $H_{\text {eqv }}$ has the same form as that of a system of free particles with equivalent mass $m_{q}$, the energy density of SQM can be expressed as

$$
E=\sum_{i=u, d, s, e} \frac{g_{i}}{2 \pi^{2}} \int_{0}^{p_{f, i}} \sqrt{p^{2}+m_{i}^{2}} p^{2} d p+B
$$

where

$$
p_{f, i}=\left(\frac{6}{g_{i}} \pi^{2} n_{i}\right)^{1 / 3}
$$

is the corresponding Fermi momentum.

As usually done, we here assume the SQM to consist of $u, d$, and $s$ quarks, and electrons (neutrinos enter and leave the system freely). The degeneracy factor $g_{i}$ is 6 for quarks and 2 for electrons. The electron mass $m_{e}$ is equal to $0.511 \mathrm{MeV}$. In order to include the strong interaction between quarks, the quark masses $m_{q}(q=u, d, s)$ should be replaced with the expression (13) or (26). The extra term $B$ comes from the pressure balance condition, and its physical meaning is still the vacuum energy density or vacuum pressure just as in the MIT bag model. The corresponding pressure is

$$
P=\sum_{i=u, d, s, e} \mu_{i} n_{i}-E,
$$

where $\mu_{i}$ is the chemical potential for particle type $i$. Because it is equal to the Fermi energy at zero temperature, we have

$$
\mu_{i}=\sqrt{p_{f, i}^{2}+m_{i}^{2}} .
$$

Equation (29) is equivalent to

$$
P=-\Omega-B .
$$

The second term $-B$ is responsible for pressure balance. Such an extra term is necessary even in the nonrelativistic treatment of SQM 15.

It is clear that the above thermodynamic treatment will approach the conventional MIT bag model if one casts away the interacting part $m_{\mathrm{I}}$ of the equivalent mass $m_{q}$. It can be proved, from Eqs. (26), (27), and (29), that we have the following correct vacuum limits:

$$
\begin{gathered}
\lim _{n_{b} \rightarrow 0} E=B, \\
\lim _{n_{b} \rightarrow 0} P=-B .
\end{gathered}
$$

Therefore, the physical meaning of $B$ is the same as that in the conventional bag model. We take $B^{1 / 4}=144 \mathrm{MeV}$ in our present calculation.

\section{PROPERTIES OF STRANGE QUARK MATTER}

Following previous authors [3], we assume the SQM to be a Fermi gas mixture of $u, d, s$ quarks and electrons with chemical equilibrium maintained by the weak interactions: $d, s \leftrightarrow u+e+\bar{\nu}_{e}, s+u \leftrightarrow u+d$. For a given baryon number density $n_{b}$ and total electric charge density $Q$, the chemical potentials $\mu_{u}, \mu_{d}, \mu_{s}$, and $\mu_{e}$ are determined by the following equations [8]:

$$
\begin{gathered}
\mu_{d}=\mu_{s} \equiv \mu, \\
\mu_{u}+\mu_{e}=\mu, \\
\frac{1}{3}\left(n_{u}+n_{d}+n_{s}\right)=n_{b}, \\
\frac{2}{3} n_{u}-\frac{1}{3} n_{d}-\frac{1}{3} n_{s}-n_{e}=Q,
\end{gathered}
$$


where the particle number density $n_{i}$ is related to the corresponding chemical potential $\mu_{i}$ by

$$
n_{i}=\frac{g_{i}}{6 \pi^{2}}\left(\mu_{i}^{2}-m_{i}^{2}\right)^{3 / 2},
$$

which is derived from the relation

$$
n_{i}=-\frac{\partial \Omega_{i}}{\partial \mu_{i}}
$$

with

$$
\begin{aligned}
\Omega_{i}=-\frac{g_{i}}{48 \pi^{2}} & {\left[\mu_{i}\left(\mu_{i}^{2}-m_{i}^{2}\right)^{1 / 2}\left(2 \mu_{i}^{2}-5 m_{i}^{2}\right)\right.} \\
& \left.+3 m_{i}^{4} \ln \frac{\mu_{i}+\sqrt{\mu_{i}^{2}-m_{i}^{2}}}{m_{i}}\right] .
\end{aligned}
$$

In order to include the strong interaction between quarks, the quark masses $m_{u}, m_{d}$, and $m_{s}$ in the above equations are to be replaced with the density-dependent expression Eq. (26) while the electron mass $m_{e}$ is negligible $(0.511 \mathrm{MeV})$.

For the bulk SQM with weak equilibrium, the previous investigations got a slightly positive charge. Our recent study demonstrates that negative charges could lower the critical density. However, too much negative charge can make it impossible to maintain flavor equilibrium. Therefore, the charge of SQM is not allowed to shift too far away from zero at both positive and negative directions. For this and our methodological purpose, we only consider neutral SQM in this paper, i.e., $Q=0$ in Eq. (37).

Since the baryonic matter is known to exist in the hadronic phase, we must require $D$ to be such that the $u d$ system is unbound. This constrains $D$ to be bigger than $(47 \mathrm{MeV})^{2}$, i.e., at $P=0, E / n_{b}>930$ in order not to contradict standard nuclear physics. On the other hand, we are interested in the possibility that SQM might be absolutely stable, i.e., at $P=0, E / n_{b}<930$, which gives an upper bound $(128 \mathrm{MeV})^{2}$. we take $D^{1 / 2}$ to be 50,80 , and $110 \mathrm{MeV}$, respectively.

Because the light quark current masses are very small, their value uncertainties are not important. So we take the fixed central values $m_{u 0}=5 \mathrm{MeV}$ and $m_{d 0}=10$ $\mathrm{MeV}$ in our calculation. As for $s$ quarks, we take 150 , 120 , and $90 \mathrm{MeV}$, corresponding respectively to $D^{1 / 2}=$ 50, 80, and $110 \mathrm{MeV}$.

For a given $n_{b}$, we first solve for $\mu_{i}(i=u, d, s, e)$ from the equation group (34)-(37), and then calculate the energy density and pressure of SQM from Eqs. (27) and (29):

$$
\begin{gathered}
E=\sum_{i} \frac{g_{i} m_{i}^{4} x_{i}^{3}}{6 \pi^{2}} F\left(x_{i}\right)+B=\sum_{i} m_{i} n_{i} F\left(x_{i}\right)+B, \\
P=\sum_{i} \frac{g_{i} m_{i}^{4} x_{i}^{5}}{6 \pi^{2}} G\left(x_{i}\right)-B=\sum_{i} m_{i} n_{i} x_{i}^{2} G\left(x_{i}\right)-B,
\end{gathered}
$$

where the summation goes over $u, d, s$, and $e$, and

$$
x_{i} \equiv \frac{p_{f, i}}{m_{i}}=\frac{\sqrt{\mu_{i}^{2}-m_{i}^{2}}}{m_{i}}
$$

is the ratio of the Fermi momentum to the mass that related to particle type $i$. With the hyperbolic sine function $\operatorname{sh}^{-1}(x) \equiv \ln \left(x+\sqrt{x^{2}+1}\right)$, the functions $F(x)$ and $G(x)$ are defined as

$$
\begin{gathered}
F(x) \equiv \frac{3}{8}\left[x \sqrt{x^{2}+1}\left(2 x^{2}+1\right)-\operatorname{sh}^{-1}(x)\right] / x^{3}, \\
G(x) \equiv \frac{1}{8}\left[x \sqrt{x^{2}+1}\left(2 x^{2}-3\right)+3 \operatorname{sh}^{-1}(x)\right] / x^{5},
\end{gathered}
$$

which have the limit properties

$$
\begin{aligned}
& \lim _{x \rightarrow 0} F(x)=1, \\
& \lim _{x \rightarrow 0} G(x)=\frac{1}{5} .
\end{aligned}
$$

Therefore, we have the correct limits Eqs. (32) and (33).

In Fig. 1, we give the energy per baryon vs baryon number density for the three pairs of parameters. We see that SQM is absolutely stable for the first two parameter groups, while metastable for the third group. The points marked with a circle " $\bigcirc$ " are the zero pressure points where the pressure within SQM is zero. Because of the density dependence of quark masses, the zero pressure density is generally not that corresponding to the minimum energy per baryon (as in the usual case), but nearly the case in the first two parameter groups.

The resulting equation of state is plotted in Fig. 2. Because it is insensitive to parameters, we have only chosen one parameter pair: $D=(80 \mathrm{MeV})^{2}$ and $m_{s 0}=120$ $\mathrm{MeV}$.

In Fig. 3, we show the sound velocity $c$ of SQM with a dot-dashed line, which is obtained from

$$
c=\left|\frac{d P}{d E}\right|^{1 / 2} .
$$

Because the interacting part of the quark masses is negligible at higher densities, it asymptotically tends to the ultrarelativistic value $1 / \sqrt{3}$ as in the bag model (solid line). Simultaneously given is that calculated by the same method as in Ref. [12] with $C=90 \mathrm{MeV} \mathrm{fm}^{-3}$ and $m_{s 0}=80 \mathrm{MeV}$ (dotted line). Obviously, the lower density behavior of the sound velocity in our model is opposite to that in the previous calculation.

It is interesting to note that if one considers the thermodynamic relation $P=-\partial(\Omega V) / \partial V$ as being more fundamental than $P=-\Omega$ (as done in Ref. 12]), Eqs. (41) and (42) should be replaced with

$$
\begin{gathered}
E=\sum_{i} m_{i} n_{i} F\left(x_{i}\right)+\sum_{i} m_{i} n_{i} f\left(x_{i}\right)+B, \\
P=\sum_{i} m_{i} n_{i} x_{i}^{2} G\left(x_{i}\right)-\sum_{i} m_{i} n_{i} f\left(x_{i}\right)-B,
\end{gathered}
$$

where

$$
f\left(x_{i}\right) \equiv-\frac{3}{2} \frac{n_{b}}{m_{i}} \frac{d m_{i}}{d n_{b}}\left[x_{i} \sqrt{x_{i}^{2}+1}-\mathrm{sh}^{-1}\left(x_{i}\right)\right] / x_{i}^{3},
$$

which has the limit property 


$$
\lim _{n_{b} \rightarrow 0} f\left(x_{i}\right)=z
$$

However, the modification does not change the properties of SQM significantly this time. For the same parameters, the line in Fig. 1 will move upward slightly while in Fig. 2 and Fig. 3 a little downward. This is because the contribution from the extra term $\sum_{i} m_{i} n_{i} f\left(x_{i}\right)$ (arising from the density dependence of the quark masses) is positive to energy and negative to pressure. But the gross features of SQM are still the same.

\section{SUMMARY}

We have presented a new version of the quark massdensity-dependent model for SQM. We first note that the previous treatments have unreasonable vacuum limits. Then we provide a practical method to derive the quark mass formulas. In our thermodynamic treatment, the conventional bag model is included as an extreme, and the vacuum still has a constant energy density corresponding to a constant pressure $B$. In this new treatment, SQM also has the possibility of absolute stability for a wide range of parameters. A noticeable feature is that the sound velocity is smaller than the ultrarelativistic case at lower densities, contrary to the previous finding.

\section{ACKNOWLEDGMENTS}

The authors would like to thank the National Natural Science Foundation of China for partial financial support.

[1] E. Witten, Phys. Rev. D 30, 272 (1984).

[2] L.M. Zhou, G.X. Peng, and P.Z. Ning, Prog. Phys. 19, 59 (1999).

[3] E. Farhi and R.L. Jaffe, Phys. Rev. D 30, 2379 (1984); M.S. Berger and R. L. Jaffe, Phys. Rev. C 35, 213 (1987); E.P. Gilson and R. L. Jaffe, Phys. Rev. Lett. 71, 332 (1993).

[4] Jes Madsen, Phys. Rev. Lett. 61, 2909 (1993); Phys. Rev. D 47, 5156 (1993); 50, 3328 (1994); Jes Madsen, Dan M. Jensen, and Michael B. Christiansen, Phys. Rev. C 53, 1883 (1996).

[5] B.C. Parija, Phys. Rev. C 48, 2483 (1993); 51, 1473 (1995).

[6] Jürgen Schaffner-Bielich, Carsten Greiner, Alexander Diener, and Horst Stöcker, Phys. Rev. D 55, 3038 (1997).

[7] G.X. Peng, P.Z. Ning, and H.C. Chiang, Phys. Rev. C 56, 491 (1997).

[8] G.X. Peng, H.C. Chiang, P.Z. Ning, and B.S. Zou, Phys. Rev. C 59, 3452 (1999).

[9] S. Chakrabarty, S. Raha, and B. Sinha, Phys. Lett. B 229, 112 (1989).
[10] S. Chakrabaty, Phys. Rev. D 43, 627 (1991); 48, 1409 (1993); 54, 1306 (1996).

[11] G.N. Fowler, S. Raha, and R.M. Weiner, Z. Phys. C 9, 271 (1981).

[12] O.G. Benvenuto and G. Lugones, Phys. Rev. D 51, 1989 (1995); G. Lugones and O.G. Benvenuto, ibid. 52, 1276 (1995).

[13] V.M. Belyaev and Ya.I. Kogan, Phys. Lett. B 136, 273 (1984); K.D. Born, E. Laermann, N. Pirch, T.F. Walsh, and P.M. Zerwas, Phys. Rev. D 40, 1653 (1989).

[14] N. Isgur and J. Paton, Phys. Lett. B 124, 273 (1983); Phys. Rev. D 31, 2910 (1985).

[15] L. Satpathy, P.K. Sahu, and V.S. Uma Maheswari, Phys. Rev. D 49, 4642 (1994).

FIG. 1. The energy per baryon vs baryon number density for different parameters. The zero pressure density occurs at the points marked with circle " $\bigcirc$."

FIG. 2. The equation of state for parameter group $D^{1 / 2}=$ $80 \mathrm{MeV}$ and $m_{s 0}=120 \mathrm{MeV}$. It asymptotically approaches to the ultrarelativistic case as expected.

FIG. 3. The sound velocity vs energy density. The dot-dashed line is calculated with the method in this paper, while the dotted line is calculated with the same method in Ref. 12]. Their lower density behavior is obviously opposite. The full line is the ultrarelativistic case. 


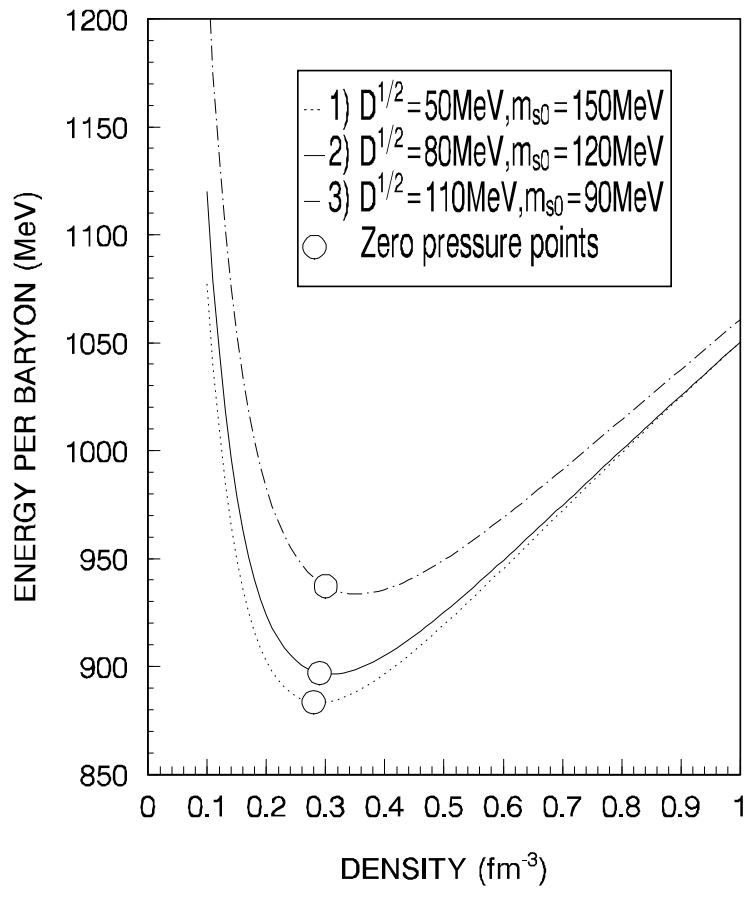




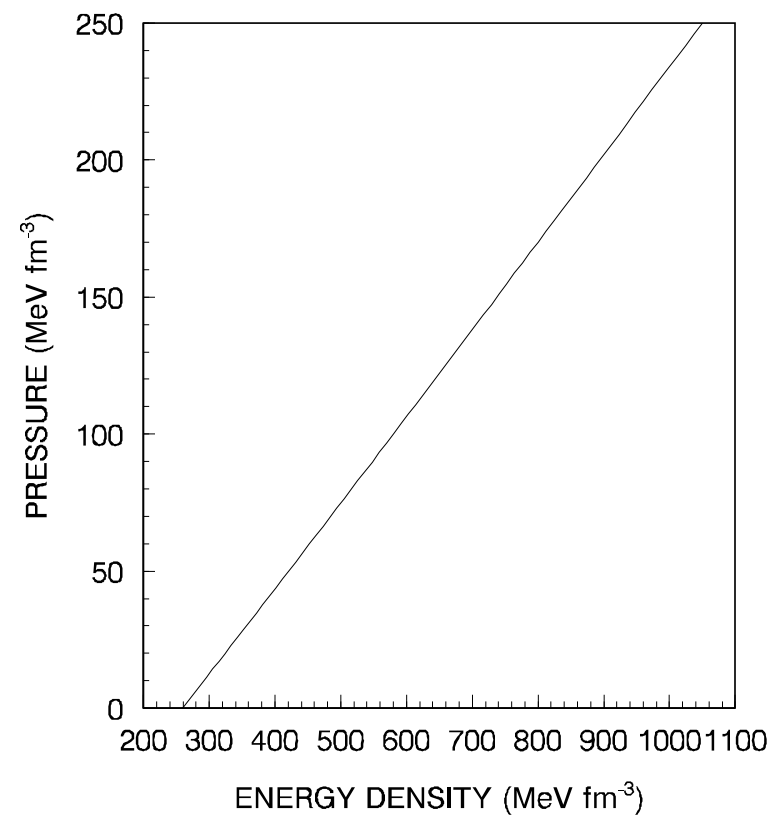




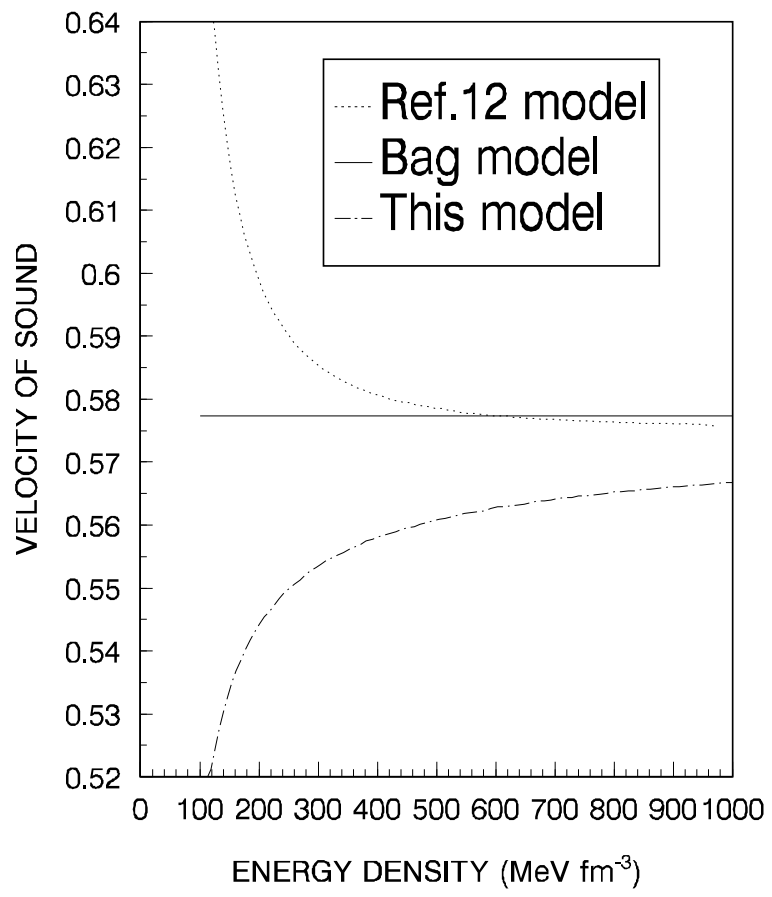

